\title{
Recherches éco-parasitologiques sur l'helminthofaune des Chiroptères dans l'est des Pyrénées
}

\author{
par Claude COMBES et Bernadette CLERC
}

Laboratoire de Zoologie et Biologie animale, Collège scientifique universitaire, $F$ 66-Perpignan

\begin{abstract}
Résumé
Les auteurs étudient l'helminthofaune de 10 espèces de Chiroptères, dont 3 avec des effectifs de plus de 70 individus : $R$. ferrum-equinum, $R$. euryale, $M$. schreibersi. Neuf Trématodes, un Cestode et trois Nématodes sont recensés.

Les principaux résultats sont les suivants :

- il existe des différences qualitatives dans l'helminthofaune des espèces de Chauves-souris, notamment pour les Nématodes;

- il existe des différences quantitatives importantes: $R$. ferrum-equinum est beaucoup plus parasité que $R$. euryale;

- il n'existe pas de différences nettes suivant le biotope de capture et suivant la saison.
\end{abstract}

\section{Summary}

The helminths of 10 species of Chiroptera have been studied, among them, three have been studied on groups of over 70 animals ( $R$. ferrum-equinum, $R$. euryale, $M$. schreibersi). Nine Trematoda, one Cestoda and three Nematoda have been found.

The main results are as follows:

- there are qualitative differences in the helminthofauna, according to the different species of bats, especially among the Nematoda ;

- there are important quantitative differences in the infestation: there are more parasites in $R$. ferrum-equinum than in R. euryale; 
- neither the biotope in which the bats have been collected, nor the season make any actual difference in the nature of the helminthofauna.

Les endoparasites des Chiroptères de France sont actuellement très mal connus puisque les travaux récents qui les concernent sont au nombre de trois seulement: Théodoridès (1953) a étudié les Helminthes de Miniopterus schreibersi Natterer chez une population cavernicole des Pyrénées-Orientales. Dollfus (1961) a dressé le catalogue des parasites de Chauves-Souris récoltés dans la région de Richelieu (Indre-et-Loire). Timon-David (1964) a étudié les Helminthes de Rhinolophus ferrum-equinum (Schreber) en Provence. Les travaux donnant un aspect quantitatif à l'étude de l'helminthofaune des Chiroptères concernent donc deux espèces d'hôtes seulement, le Minioptère de Schreiber et le grand Rhinolophe fer-à-cheval. Nous ajouterons que si, dans d'autres pays, de nombreux travaux ont abordé les aspects systématique ou faunistique du problème, des études statistiques portant sur des populations déterminées et débouchant sur des corrélations de nature écologique n'ont pas été entreprises. Seul, le travail de Mituch (1964), concernant les Rhinolophidés de Tchécoslovaquie, fournit une comparaison détaillée entre les helminthofaunes de trois espèces d'hôtes, examinés en quantités satisfaisantes. Cependant Holmes (1966) a souligné l'intérêt des Trématodes de ChauvesSouris, dont la plupart sont spécifiques de ces Mammifères et peuvent fournir des indications sur le régime et le déplacement de leurs hôtes.

Nous avons effectué, au cours des années 1967 et 1968, l'examen parasitologique de 293 Chiroptères appartenant à dix espèces différentes, capturés dans plusieurs localités des Pyrénées-Orientales (fig. 1). Nous donnons la liste de ces espèces avec, entre parenthèses, le nombre d'animaux examinés de chacune d'elles (1):

Rhinolophidés :

- Rhinolophus ferrum-equinum (Schreber)

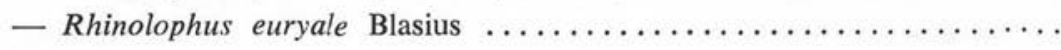

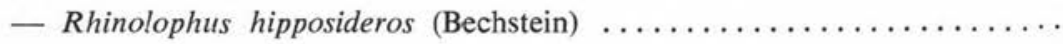

Vespertilionidés :

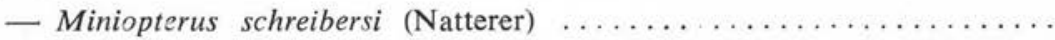

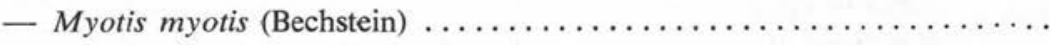

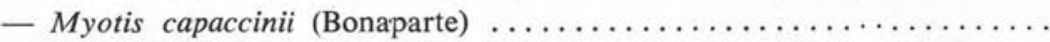

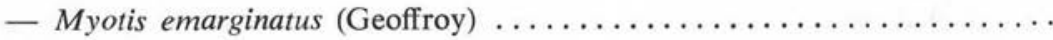

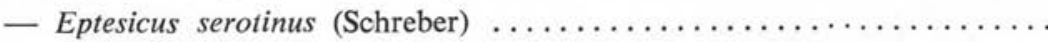

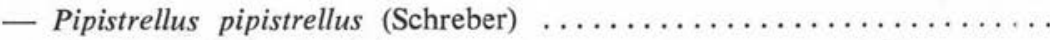

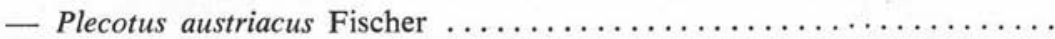

(1) Nous remercions vivement M. Henri Salvayre, du Groupe d'Etudes et de Recherches Spéléologiques du C.S.U. de Perpignan, dont la connaissance des milieux souterrains et l'aide qu'il n'a cessé de nous apporter ont grandement facilité la réalisation de ce travail.

Nous exprimons notre reconnaissance à M. Georges Dubois, de l'Université de Neuchâtel, qui a bien voulu vérifier la plupart des déterminations de Trématodes Lecithodendriidae. 
On voit que nos recherches les plus approfondies concernent le Grand Rhinolophe fer-à-cheval, le Rhinolophe euryale et le Minioptère de Sohreiber.

Le seul organe nous ayant livré des Helminthes a été en règle générale le tube digestif, des Filaires ayant été cependant rencontrées dans la cavité générale.

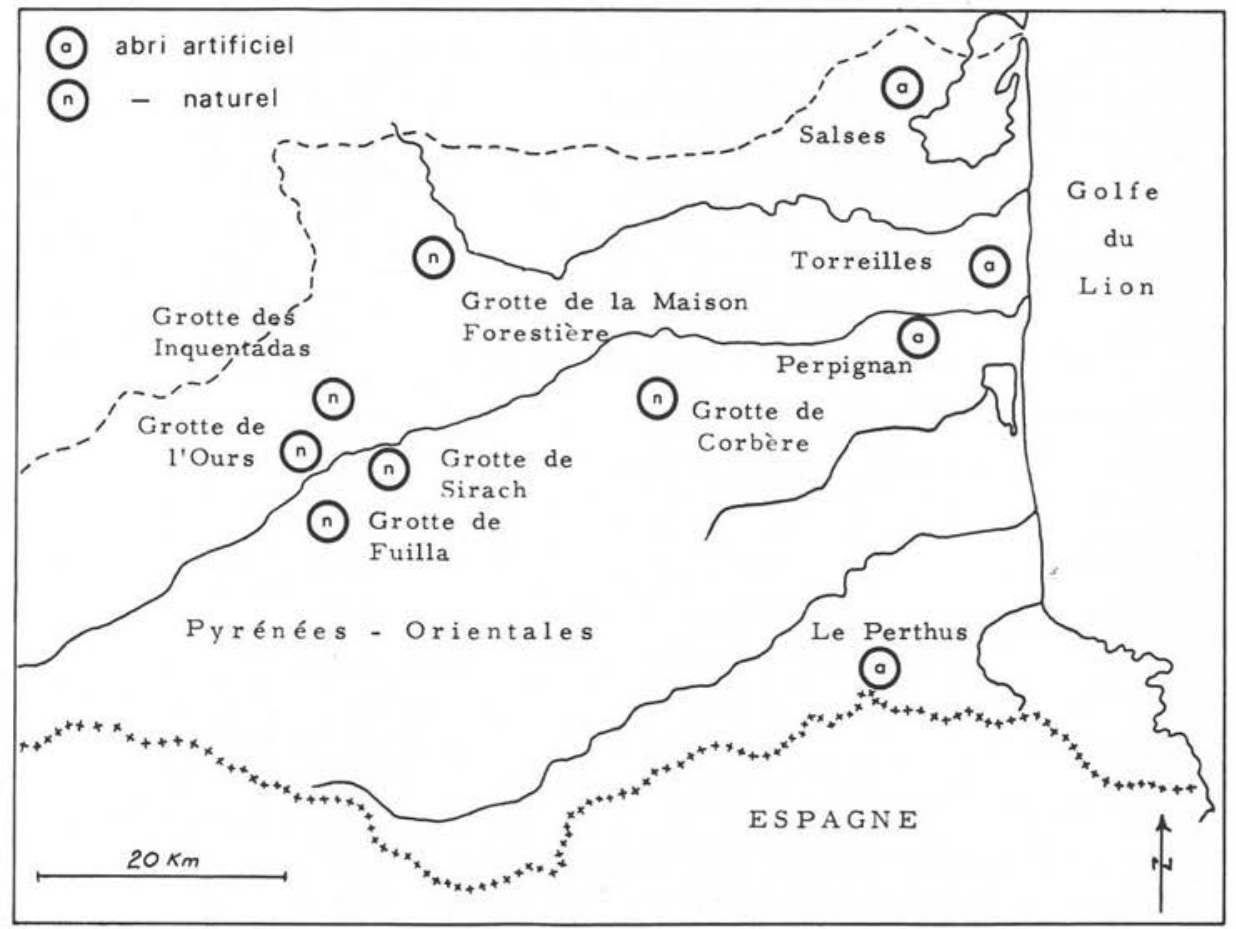

F1G. 1. - Localités prospectées. CoordonnéEs: $G$. de Corbère: EM Prades 1/50.000, $620,150 \times 39,250 \mathrm{Z} 270 \mathrm{~m}-G$. de Fuilla: idem $601,625 \times 30,975 \mathrm{Z} 475 \mathrm{~m}-$ G. de l'Ours: idem $602,650 \times 31,750 \mathrm{Z} 510 \mathrm{~m}-G$. de Sirach: idem $605,625 \times 33,200 \mathrm{Z} 490 \mathrm{~m}-G$. des Inquentadas: F.M St-Paul-de-Fenouillet 1/50.000, 597,100 × 41,925 Z $900 \mathrm{~m}-$ G. de la Maison Forestière: idem $611,800 \times 47,150$ Z $390 \mathrm{~m}-$ Perpignan: EM Perpignan, $647,200 \times 44,500 \mathrm{Z} 55 \mathrm{~m}-$ Salses: iaem $647,550 \times 60,150 \mathrm{Z} 10 \mathrm{~m}$ - Torreilles: idem $653,750 \times 50,975 \mathrm{Z} 5 \mathrm{~m}-$ Le Perthus: EM Arles-sur-Tech, $643,000 \times 17,750 \mathrm{Z} 400 \mathrm{~m}$.

Nous avons identifié les Trématodes Digènes, les Cestodes et les Nématodes suivants :

a) Trématodes Digènes (1):

LECITHODENDRIIDAE Odhner, 1910.

Lecithodendriinae Looss, 1902

(1) Pour tous les Trématodes que nous citons, Matskasi (1967 et 1968) a répertorié de manière très complète les hôtes connus et les localités géographiques signalées. Hurkova (1964) a donné une clé de détermination où prennent place également nos différentes espèces. 
Prostho 'en.lrium chiiostomum (Mehl's, 1831).

Prosthodendrium parvouterus (Bhalerao, 1926).

Lecithodendrium linstowi Dollfus, 1931.

Lecithodendrium granulosum Looss, 1907.

Pycnoporinae Yamaguti, 1958.

Pycnoporus heteroporus (Dujardin, 1845).

Parabascinae Yamaguti, 1958.

Parabascus semisquamosus (Braun, 1900).

Parabascus lepidotus Looss, 1907.

PLAGIORCHIIDAE Ward, 1917.

Plagiorchiinae Pratt, 1902.

Plagiorchis vespertilionis (Müller, 1784).

Mesotretidae Poche, 1926.

Mesotretes peregrinus (Braun, 1900).

\section{b) Cestodes :}

HYMENOLEPIDIDAE Railliet et Henry, 1909.

Hymenolepis grisea (Van Beneden, 1873).

\section{c) Nématodes :}

TRIC HOSTRONGYLIDAE Leiper, 1912.

Strongylacanthinae Yorke et Maplestone, 1926.

Strongylacantha glycirrhiza Van Beneden, 1873.

Molinostrongylus ornatus (Mönnig, 1929).

DiPeTAlonematidae Wehr, 1935.

Dipetalonematinae Wehr, 1935.

Listomosa beaucournui Bain, 1967.

Parmi les espèces citées ci-dessus, certaines sont bien connues et ne nécessitent aucune précision supplémentaire quant à leur description. C'est le cas des Trématodes Plagiorchiidae et Mesotretidae, des Cestodes et des Nématodes. Par contre, pour les Trématodes Lecithodendriidae, formes de petites dimensions et de taxonomie délicate, nous pensons qu'il est utile d'indiquer les mensurations des exemplaires recueillis au cours de nos recherches (Pour toutes nos mesures, le nombre entre parenthèses indique la dimension moyenne; lorsque deux séries de dimensions sont données pour un organe, la première concerne soit la longueur, soit le diamètre antéro-postérieur, la seconde concerne soit la largeur, soit le diamètre transversal). 
Prosthodendrium chilostomum (Mehlis, 1831), fig. 2.

Mensurations d'après cinq exemplaires recueillis chez $M$. schreibersi.

Longueur : 0,49 à $0,80 \mathrm{~mm}(0,64)$. Largeur : 0,29 à $0,40 \mathrm{~mm}(0,34)$. Ventouse orale : 154 à 200 sur 131 à $171 \mu$ (177 sur 148). Ventouse ventrale : 85 à $97 \mu(88)$. Distance entre centres des ventouses : 234 à $383 \mu$ (280). Pharynx : 37 à $57 \mu$ (41). Ovaire : 120 à 130 sur 95 à

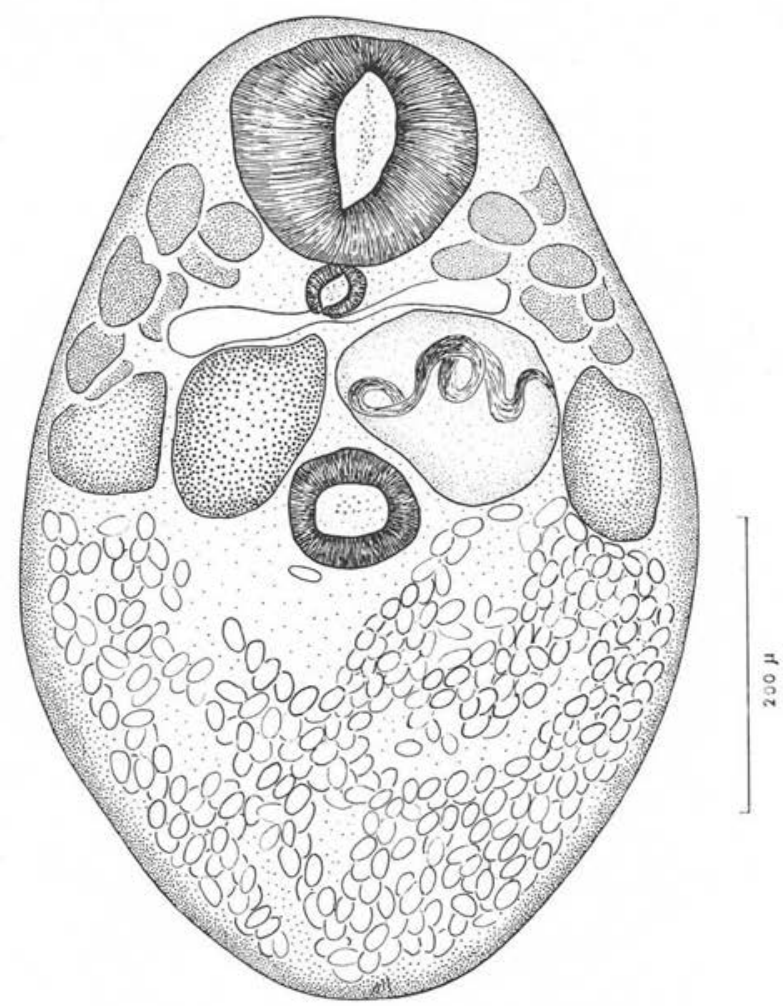

Fig. 2. - Prosthodendrium chilostomum (Melhis, 1831), exemplaire récolté chez Miniopterus schreibersi

$110 \mu$ (125 sur 103). Testicules : 85 à 115 sur 74 à $85 \mu$ (97 sur 81$)$. Masse prostatique : 110 à 118 sur 117 à $148 \mu$ (114 sur 128). CEufs : 22 à 25 sur 11 à $17 \mu(24$ sur 14).

Le rapport VO/VV est égal en moyenne à 1,82 . Le nombre de follicules vitellogènes par grappe latérale varie de 7 à 9 .

Les mensurations données par divers auteurs pour cette espèce varient dans de notables proportions (voir Dubois, 1955 , p. $480-481$; et 1960 , p. 13) ; les mesures de nos exemplaires 'se rapprochent surtout de celles données par Dubois (1955, p. 481) pour des exemplaires récoltés chez $R$. hipposideros en Suisse. 
Prosthodendrium parvouterus (Bhalerao, 1926), fig. 3.

Mensurations d'après deux exemplaires recueillis chez $M$. schreibersi.

Longueur: 0,63 à $0,66 \mathrm{~mm}(0,65)$. Largeur : 0,81 à $0,86 \mathrm{~mm}(0,84)$. Ventouse orale : 140 à 146 sur 150 à $158 \mu$ (143 sur 154). Ventouse ventrale: 111 à 130 sur 114 à $125 \mu$ (120 sur 118). Distance entre centres des ventouses: 370 à $480 \mu$ (425). Pharynx : 40 à 51 sur 51 à $57 \mu$ (45 sur 54). Ovaire: 171 à 188 sur 137 à $160 \mu$ (180 sur 149). Testicules : 171 à 228 sur 148 à $217 \mu$ (192 sur 183). Masse prostatique : 268 à 274 sur 190 à $194 \mu(271$ sur 192). CEufs : 25 à 28 sur 11 à $14 \mu$ (26 sur 13).

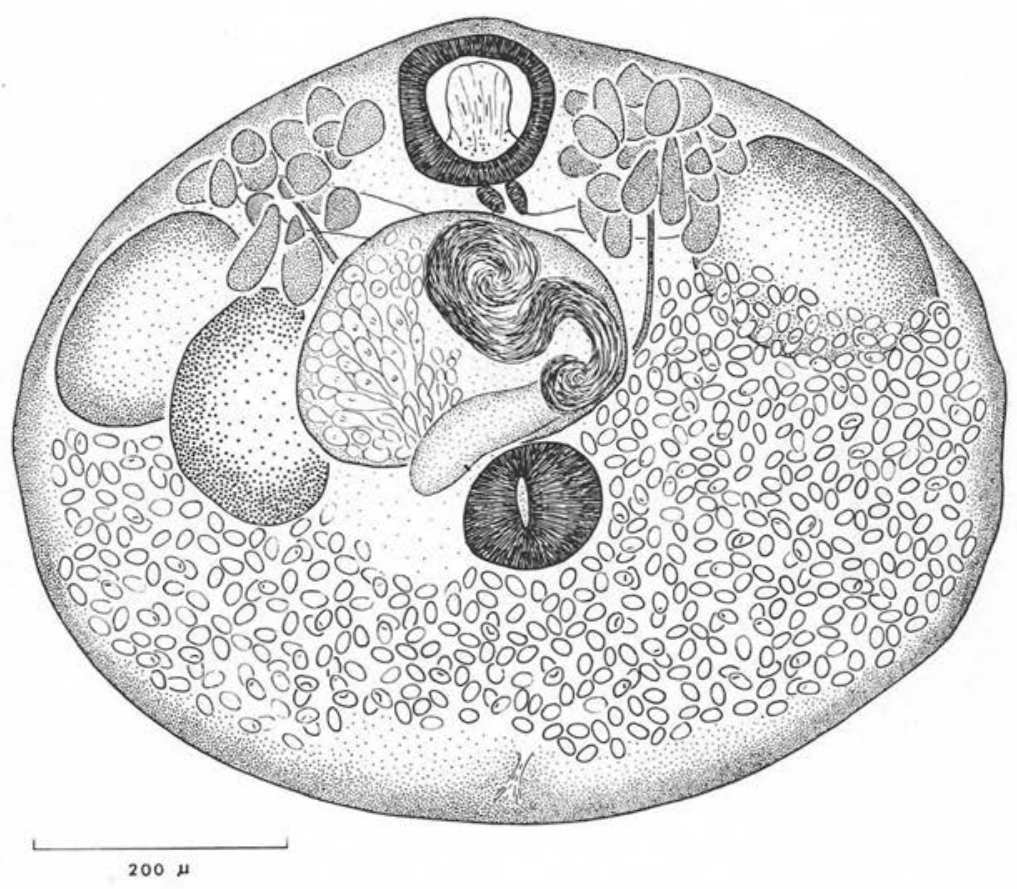

FIG. 3. - Prosthodendrium parvouterus (Bhalerao, 1926), exemplaire récolté chez Miniopterus schreibersi

Le rapport VO/VV est égal en moyenne à 1,22. Le nombre de follicules vitellogènes par grappe latérale varie de 13 à 18 .

Ces mensurations correspondent à celles données par d'autres auteurs; cependant le nombre de follicules vitellogènes par grappe est faible par rapport aux chiffres donnés par Bhalerao (1926), Dollfus (1954), Yamaguti et Asada (1942), Liang-Sheng (1957), soit pour l'espèce $P$. parvouterus elle-même, soit pour des espèces (pushpai, pyramidum, orientale, cordiforme) que Dubois (1955) considère comme synonymes de parvouterus. 
Lecithodendrium linstowi Dollfus, 1931, fig. 4.

Mensurations d'après dix exemplaires recueillis chez $R$. ferrum-equinum.

Longueur: 0,75 à $1,14 \mathrm{~mm}(1,05)$. Largeur : 0,43 à $0,68 \mathrm{~mm}(0,60)$. Ventouse orale : 540 à $910 \mu$ (77). Ventouse ventrale : 51 à $97 \mu(80)$. Distance entre centres des ventouses :

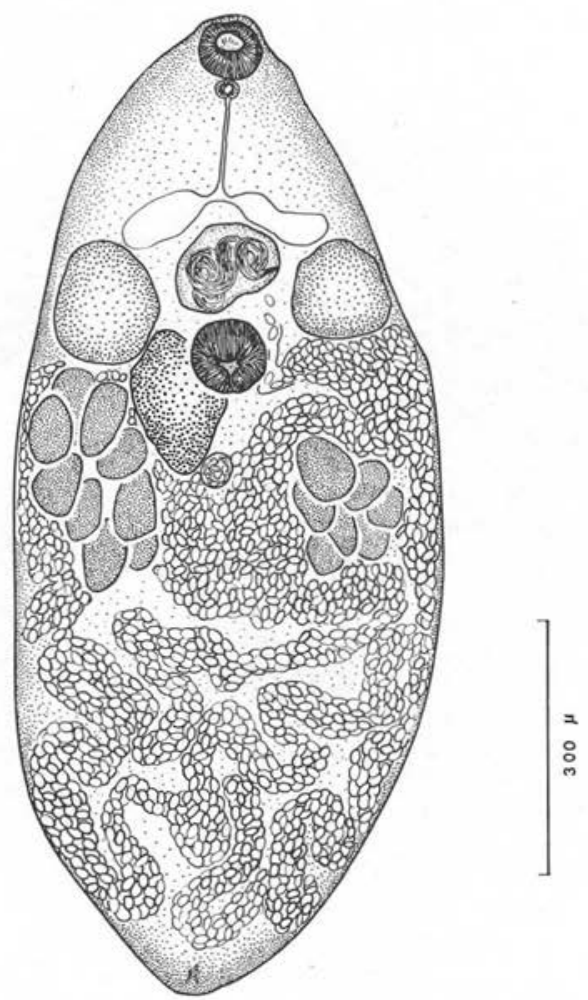

FIG. 4. - Lecithodendrium linstowi Dollfus, 1931, exemplaire récolté chez Rhinolophus ferrum-equinum (Schreber)

257 à $400 \mu$ (317). Pharynx : 28 à $34 \mu$ (31). Ovaire : 142 à 200 sur 94 à $171 \mu(157$ sur 131). Testicules: 122 à 222 sur 88 à $122 \mu$ (165 sur 115). Masse prostatique : 97 à 140 sur 91 à $117 \mu$ (120 sur 103). Eufs : 19 à 21 sur 10 à $12 \mu$ (20 sur 11).

Le rapport VO/VV est égal en moyenne à 0,96 . Le nombre de follicules vitellogènes par grappe latérale est de 9 du côté de l'ovaire et de 7 du côté opposé.

Nos exemplaires ne représentent pas de caractère original. La constance du nombre de follicules vitellogènes a été signalée par Dubois (1960), Hurkova (1963), Timon-David (1964) et Matskasi (1967). 
Lecithodendrium granulosum Looss, 1907, fig. 5.

Mensurations d'après 10 exemplaires recueillis chez $M$. capaccinii.

Longueur : 0,27 à $0,39 \mathrm{~mm}(0,31)$. Largeur : 0,18 à $0,40 \mathrm{~mm}(0,27)$. Ventouse orale : 48 à 62 sur 45 à $60 \mu$ (56 sur 53). Ventouse ventrale : 45 à 88 sur 42 à $72 \mu(62$ sur 60). Distance entre centres des ventouses : 120 à $167 \mu$ (150). Pharynx : 20 à $22 \mu$ (21). Ovaire : 57 à 68 sur 48 à $62 \mu$ ( 59 sur 55$)$. Testicules : 54 à 100 sur 42 à $96 \mu$ (77 sur 64). Masse prostatique : 60 à 105 sur 45 à $100 \mu$ ( 75 sur 68). CEufs : 17 à 20 sur 8 à $11 \mu(18$ sur 10$)$.

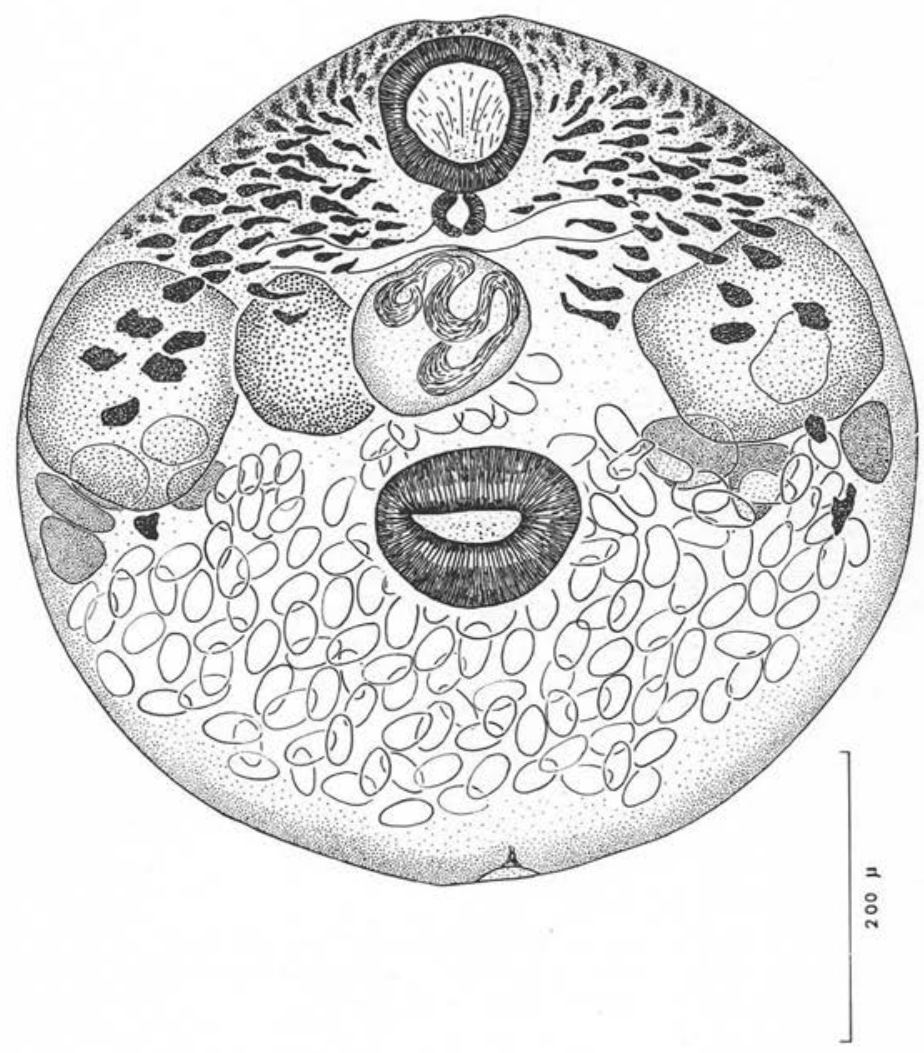

FIG, 5. - Lecithodendium granulosum Looss, 1907, exemplaire récolté chez Myotis capaccinii

Le rapport VO/VV est égal en moyenne à 0,88 . Les vitellogènes sont formés de deux grappes para et post-testiculaires, comprenant quelques gros follicules constitués de masses plus petites juxtaposées, difficiles à dénombrer.

Toute la partie antérieure de ce Digène extrêmement petit est obscurcie par de très nombreuses cellules glandulaires plus ou moins piriformes et fortement colorables. 
Pycnoporus heteroporus (Dujardin, 1845), fig. 6.

Mensurations d'après un exemplaire recueilli chez $P$. pipistrellus.

Longueur: $0,91 \mathrm{~mm}$. Largeur : $0,35 \mathrm{~mm}$. Ventouse orale : 57 sur $51 \mu$. Ventouse ventrale : 200 sur $274 \mu$. Distance entre centres des ventouses : $343 \mu$. Pharynx : 28 sur $25 \mu$. Ovaire : 134 sur $82 \mu$. Testicules : 171 sur 120 et 200 sur $108 \mu$. Masse prostatique : 111 sur $85 \mu$. Eufs : 17 à 20 sur $8 \mu$.

Le rapport VO/VV est égal à 0,22 . Le nombre des follicules vitellogènes est de 8 côté ovaire et de 7 côté opposé.

Notre exemplaire présente un rapport ventousaire particulièrement réduit (on peut calculer un rapport de 0,26 d'après les chiffres de Matskasi, 1967).

Parabascus semisquamosus (Braun, 1900), fig. 7.

Mensurations d'après cinq exemplaires recueillis chez $P$. pipistrellus.

Longueur: 0,91 à $1,53 \mathrm{~mm}(1,22)$. Largeur : 0,31 à $0,37 \mathrm{~mm}(0,34)$. Ventouse orale : 51 à $80 \mu$ (63). Ventouse ventrale : 80 à $97 \mu(87)$. Distance entre centres des ventouses : 270 à $584 \mu$ (448). Pharynx : 17 à 28 sur 23 à $28 \mu$ (26 sur 24). Ovaire: 114 à 165 sur 91 à $160 \mu(128$ sur 119$)$. Testicule antérieur : 137 à 211 sur 131 à $160 \mu(177$ sur 142). Testicule postérieur: 143 à 165 sur 114 à $142 \mu$ (154 sur 125). Masse prostatique : 148 à 188 sur 68 à $94 \mu$ (168 sur 76). Eufs: 17 à 22 sur 11 à $24 \mu$ (20 sur 12).

Le rapport VO/VV est égal en moyenne à 0,72 . Le nombre de follicules vitellogènes par grappe latérale varie de 11 à 15 .

Nos exemplaires présentent des dimensions relativement modestes par rapport à celles indiquées par Hurkova (1964) et Matskasi (1967).

Parasbascus lepidotus Looss, 1907, fig. 8.

Mensurations d'après dix exemplaires recueillis chez $M$. schreibersi.

Longueur: 0,56 à $1,14 \mathrm{~mm}(0,82)$. Largeur : 0,34 à $0,65 \mathrm{~mm}(0,47)$. Ventouse orale : 45 à $97 \mu(65)$. Ventouse ventrale : 62 à 134 sur 57 à $131 \mu(120$ sur 85$)$. Distance entre centres des ventouses : 340 à $500 \mu$ (412). Pharynx : 28 à $40 \mu$ (32). Ovaire : 117 à 171 sur 65 à $100 \mu(135$ sur 82$)$. Testicules : 85 à 228 sur 91 à $225 \mu$ (150 sur 137). Masse prostatique : 160 à 200 sur 66 à $85 \mu$ (180 sur 75$)$. Eufs : 18 à 21 sur 11 à $12 \mu$ (20 sur 11).

Le rapport VO/VV est égal en moyenne à 0,75 . Le nombre de follicules vitellogènes par grappe latérale varie de 20 à 27 .

Ces mensurations sont très semblables à celles données par d'autres auteurs. Contrairement à ceux de l'espèce précédente, nos exemplaires doivent être considérés cependant comme de taille plutôt grande. 


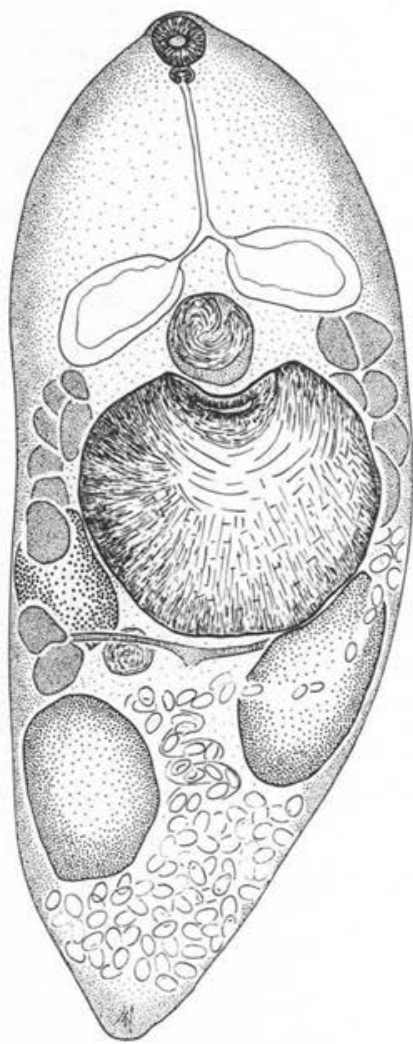

FIG. 6. - Pycnoporus heteroporus (Dujardin, 1845), exemplaire récolté chez Pipistrellus pipistrellus

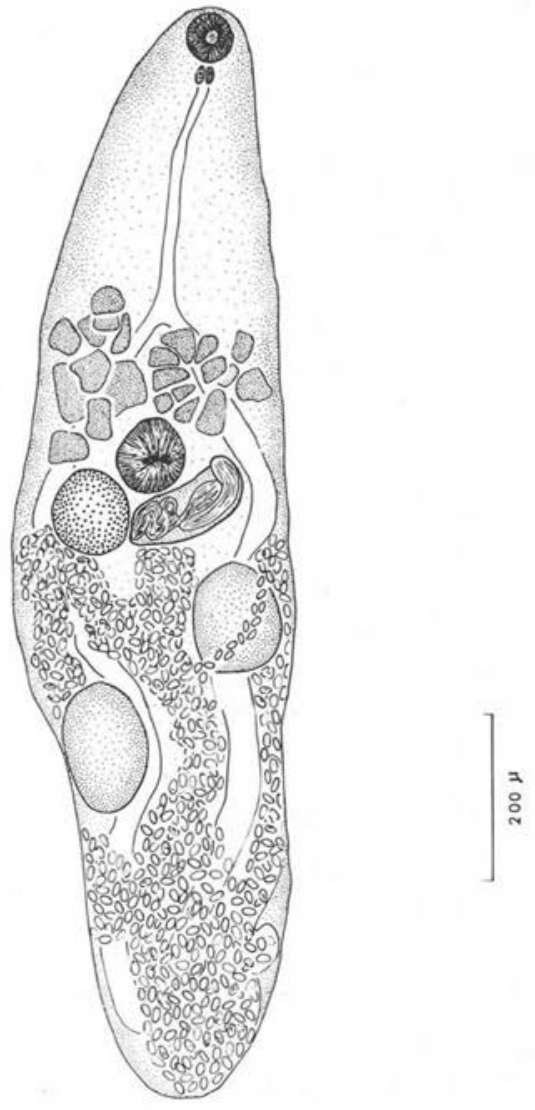

F1G. 7. - Parabascus semisquamosus (Braun, 1900), exemplaire récolté chez Pipistrellus pipistrellus

\section{Caractéristiques de l'infestation en fonction de l'hôte}

Nous avons pu mettre en évidence, suivant l'espèce d'hôte, des différences qualitatives ou quantitatives. Ces dernières sont particulièrement intéressantes à noter, car elles peuvent traduire, soit une immunité partielle de l'hôte à l'échelle de la population, soit un comportement différent de l'espèce; dans les deux cas, elles permettent de définir les hôtes comme plus ou moins favorables au développement de tel ou tel parasite et par-là même d'apprécier leur rôle dans l'écologie des cycles. 


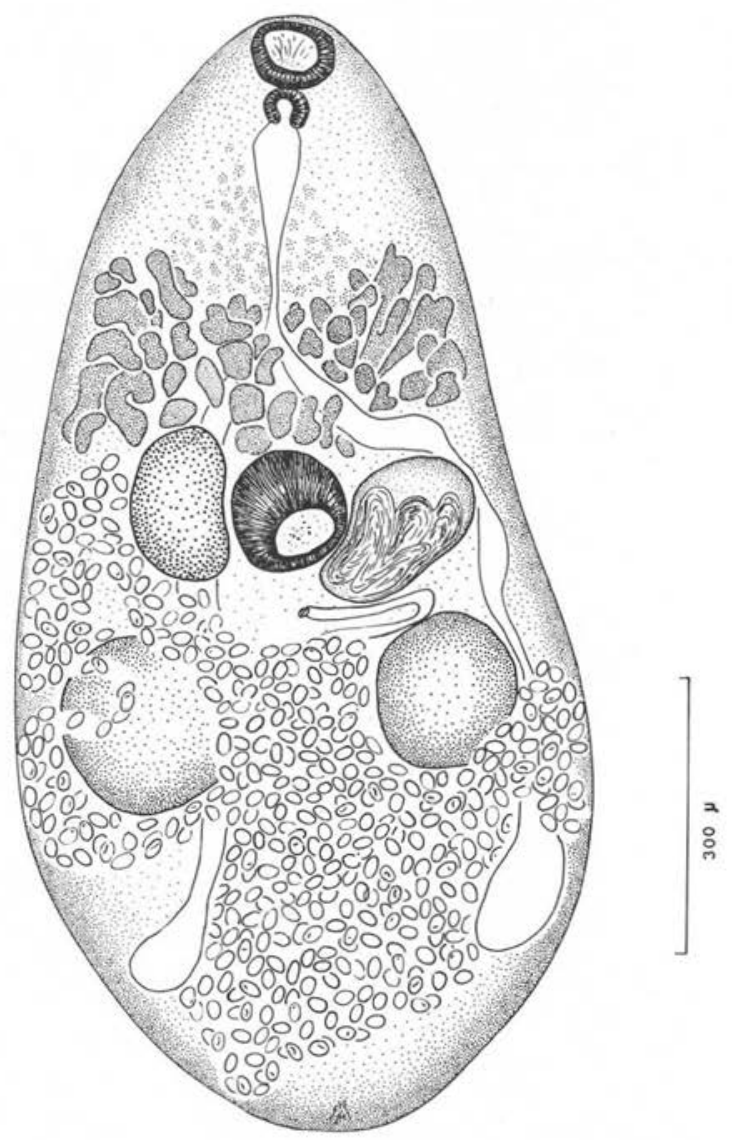

FIG. 8. - Parabascus lepidotus Looss, 1907, exemplaire récolté chez Miniopterus schreibersi

Cette étude comparative a été faite principalement pour les trois espèces de Chiroptères dont les effectifs examinés sont importants : $R$. ferrum-equinum, $R$. euryale, $M$. schreibersi. Les tableaux I, II et III résument des prélèvements-types de ces trois espèces, réalisés en des lieux peu éloignés et à des dates voisines. Le tableau IV indique les fréquences et les densités moyennes des parasites pour l'ensemble des prélèvements. Pour ces tableaux et les suivants, les abréviations utilisées sont:

P. ve. $=$ Plagiorchis vespertilionis. $M$. pe. $=$ Mesotretes peregrinus $L$. li. $=$ Lecithodendrium linstowi. $P . p a=$ Prosthodendrium parvouterus. $P$. ch. $=$ Prosthodendrium chilostomum. $P$. le. $=$ Parabascus lepidotus. $H . g r .=$ Hymenolepis grisea. $S$. gl. $=$ Strongylacantha glycirrhiza. M. or. $=$ Molinostrongylus ornatus. L. be. $=$ Litomosa beaucournui. Trém. $=$ Trématodes. Cest. $=$ Cestodes. Ném. $=$ Nématodes. 
Tableau I

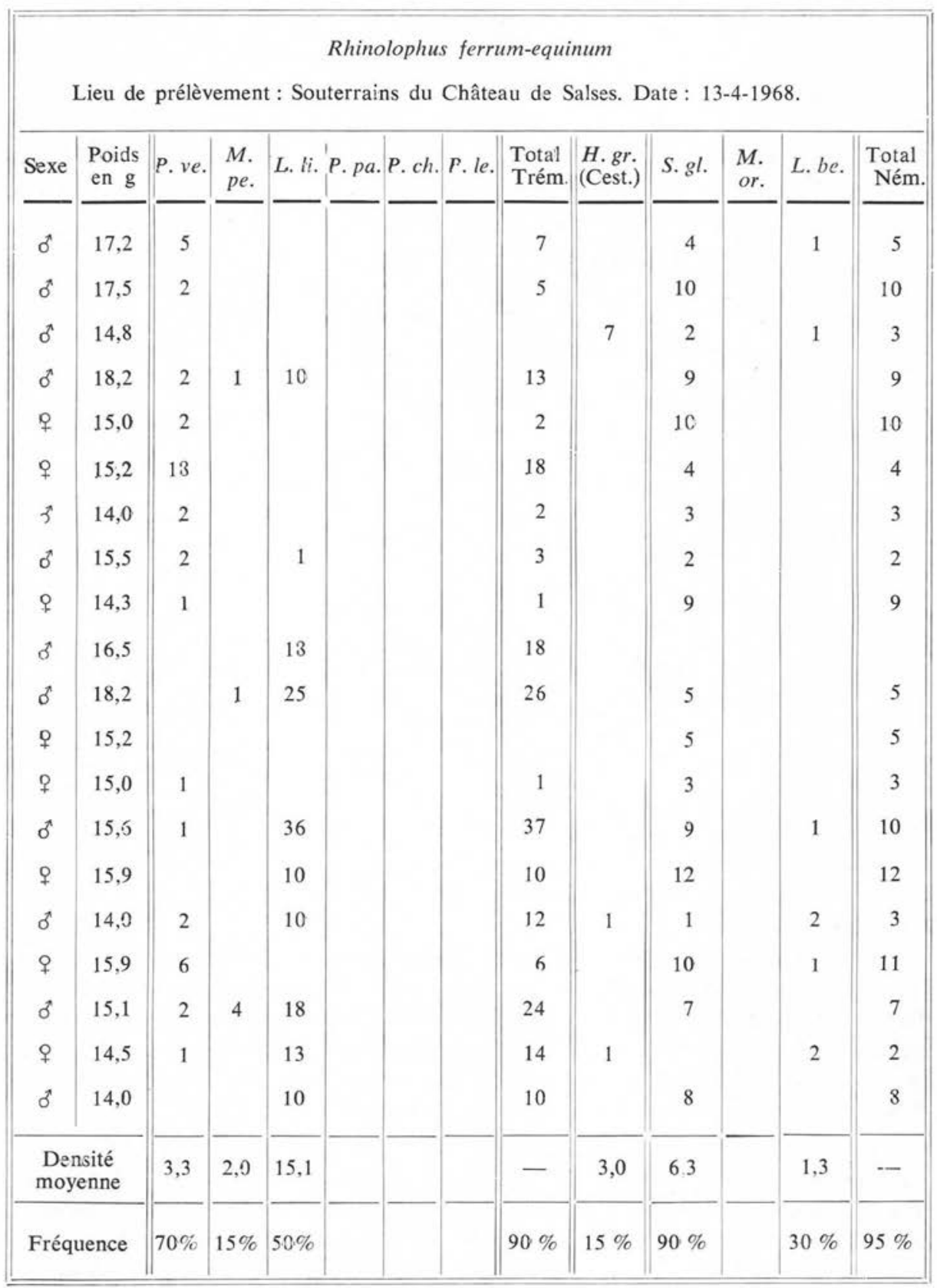


Tableau II

\section{Rhinolophus euryale}

Lieu de prélèvement: Grotte de Fuilla. Date : 8-5-1968.

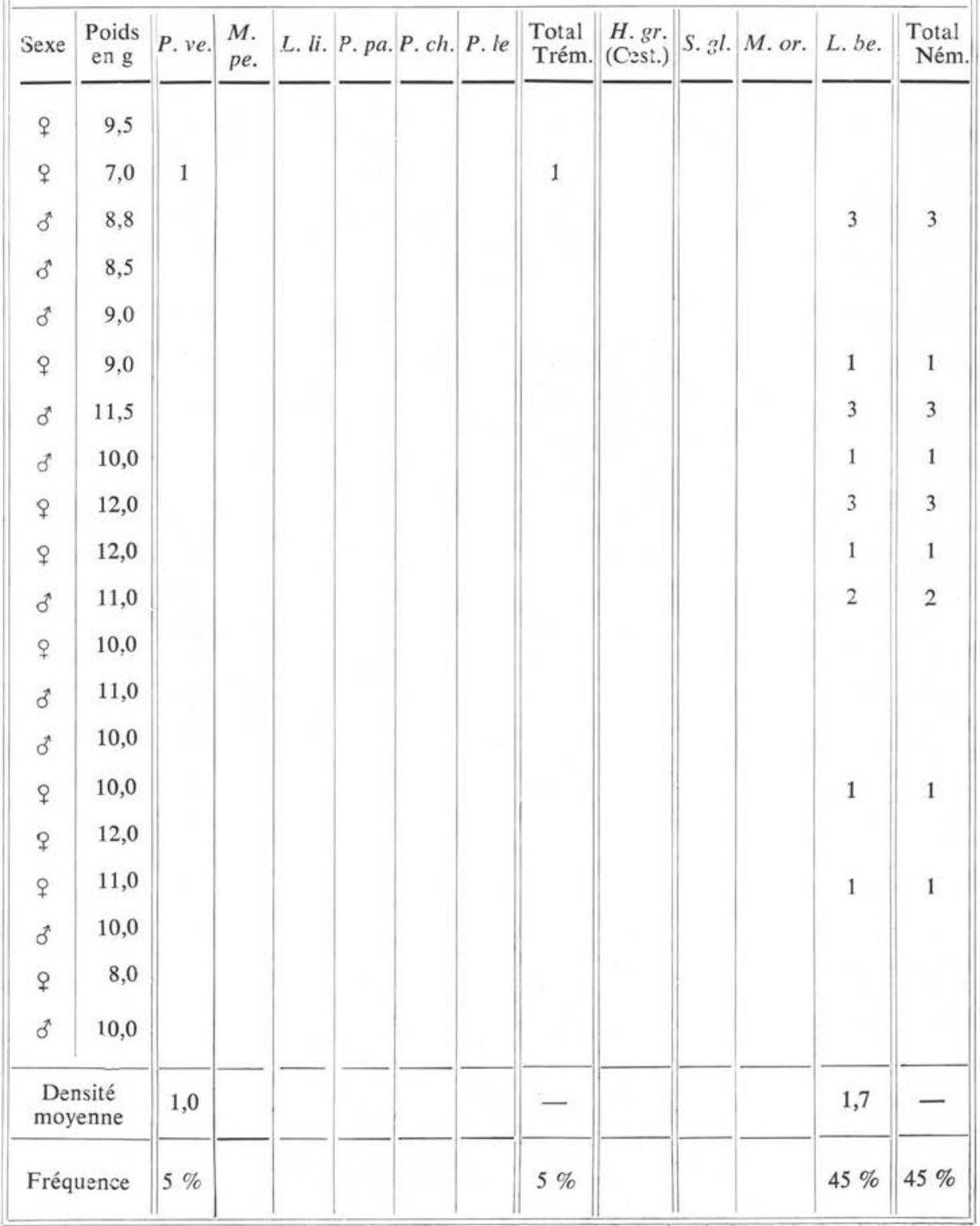


C. COMBES et B. CLERC

Tableau III

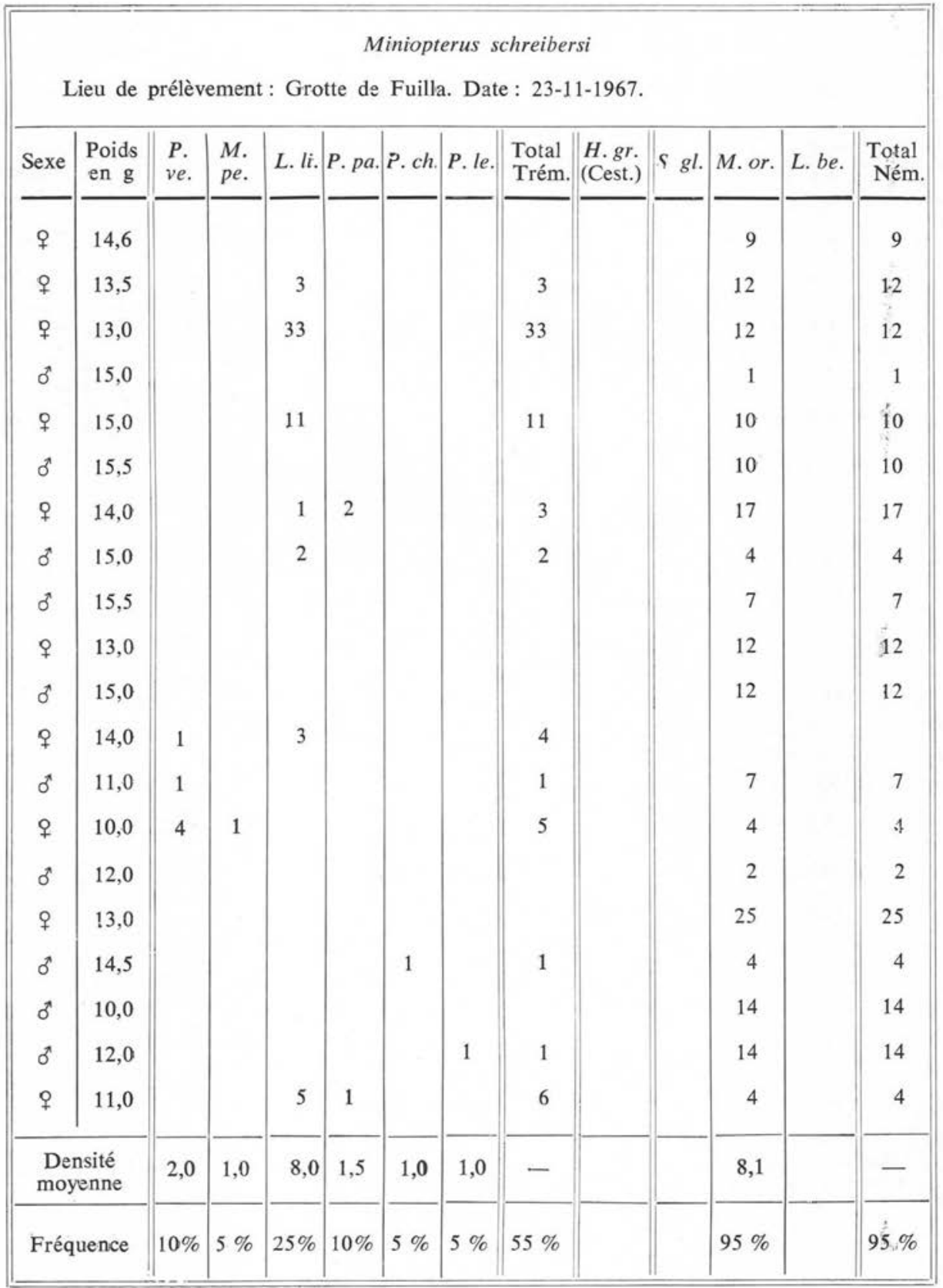




\begin{tabular}{|c|c|c|c|c|c|c|}
\hline 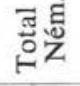 & 1 & $\stackrel{20}{\infty}$ & I & $\begin{array}{l}\Delta \\
2\end{array}$ & 1 & $\begin{array}{l}20 \\
5\end{array}$ \\
\hline $\begin{array}{l}\dot{\Xi} \\
\dot{j}\end{array}$ & $N$ & $\begin{array}{l}\Delta \\
\pm\end{array}$ & $I$ & $\begin{array}{l}\Delta \\
\Delta\end{array}$ & $\vec{i}$ & $\begin{array}{l}28 \\
\infty\end{array}$ \\
\hline $\begin{array}{l}\dot{\vdots} \\
\dot{z}\end{array}$ & 1 & 0 & 1 & 0 & $\begin{array}{l}0 \\
\infty\end{array}$ & $\frac{2}{a}$ \\
\hline $\begin{array}{l}\dot{0} \\
\dot{i}\end{array}$ & $\begin{array}{l}\infty \\
\text { in }\end{array}$ & $\frac{5}{80}$ & 1 & 0 & 1 & 0 \\
\hline 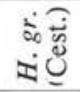 & $\stackrel{\circ}{\stackrel{\circ}{2}}$ & $\begin{array}{l}20 \\
2\end{array}$ & 1 & 0 & $\stackrel{\varrho}{=}$ & 2 \\
\hline 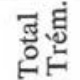 & 1 & $\begin{array}{l}20 \\
\infty\end{array}$ & 1 & $\begin{array}{l}\Delta \circ \\
\pm\end{array}$ & 1 & $\begin{array}{l}8 \\
n \\
n\end{array}$ \\
\hline$\frac{\dot{2}}{\dot{2}}$ & $\stackrel{\circ}{i}$ & $s^{\circ}$ & $\stackrel{0}{=}$ & $\stackrel{80}{m}$ & in & $8^{\circ}$ \\
\hline$\dot{0}$ & 1 & 0 & I & 0 & $\stackrel{\infty}{=}$ & $\frac{2}{1}$ \\
\hline$\frac{\dot{\Xi}}{2}$ & 1 & 0 & 1 & 0 & $\cong$ & 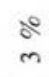 \\
\hline$=$ & $\stackrel{\text { i̊ }}{0}$ & $\stackrel{8}{\infty}$ & $\stackrel{0}{0}$ & $\begin{array}{l}80 \\
+\end{array}$ & $\stackrel{g}{ \pm}$ & $\begin{array}{l}2 \\
2\end{array}$ \\
\hline$\dot{\Sigma}$ & $\hat{i}$ & $\begin{array}{l}20 \\
2\end{array}$ & I & 0 & $\cong$ & $\begin{array}{l}\text { se } \\
\text { in }\end{array}$ \\
\hline $\begin{array}{l}\dot{2} \\
\dot{2}\end{array}$ & $\stackrel{\circ}{+}$ & $\begin{array}{l}\delta^{\circ} \\
\text { ì }\end{array}$ & i & se & $\overrightarrow{\text { तi }}$ & $\begin{array}{l}0 \\
0\end{array}$ \\
\hline & 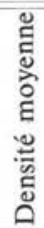 & 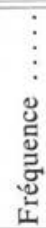 & 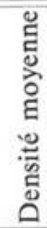 & 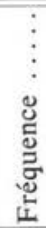 & 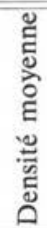 & 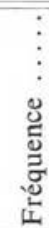 \\
\hline & \multicolumn{2}{|c|}{ 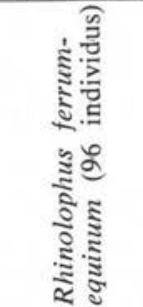 } & \multicolumn{2}{|c|}{ 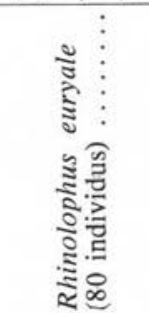 } & \multicolumn{2}{|c|}{ 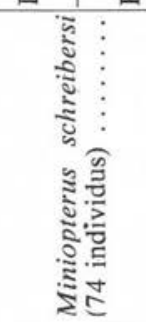 } \\
\hline
\end{tabular}




\section{Différences qualitatives et quantitatives de l'infestation chez $R$. ferrum-equinum et $R$. euryale}

Il existe des différences nettes et constantes dans le parasitisme entre $R$. ferrumequinum et $R$. euryale. Ces deux espèces de Chauves-Souris sont cependant très voisines: morphologiquement, leur distinction est délicate (rappelons qu'elles diffèrent essentiellement par la taille plus forte chez $R$. ferrum-equinum et par la forme de la lancette; celle-ci présente une dent supérieure émoussée chez $R$. ferrum-equinum, aiguë chez $R$. euryale). De plus, ces Chauves-Souris sont rencontrées dans les mêmes biotopes, parfois même mélangées lorsqu'elles sont au repos ou en hibernation.

On peut faire les observations suivantes, résumées dans la fig. 9.

\section{a) Trématodes :}

La fréquence calculée pour l'ensemble des espèces de Trámatodes s'élève à $89 \%$ chez $R$. ferrum-equinum, alors qu'elle n'est que de $14 \%$ chez $R$. euryale; la différence de densité moyenne est également remarquable: 16,2 chez $R$. ferrum-equinum, 2,6 chez $R$. euryale. Précisons que ces différences se traduisent nettement au cours des dissections : l'intestin ouvert de $R$. ferrum-equinum présente le plus souvent un aspect particulier dû à la forte densité des Trématodes et principalement des L. linstowi dans les cinq premiers centimètres; l'intestin de $R$. euryale présente au contraire un aspect sain et net.

Ces différences concernant l'ensemble des Trématodes se retrouvent lorsqu'on examine séparément chaque espèce. Ainsi, la fréquence de L. linstowi atteint $58 \%$ chez $R$. ferrum-equinum et $4 \%$ seulement chez $R$. euryale; les densités moyennes de cette espèce varient dans le même sens : 20,6 et 4,6. Les résultats sont très semblables pour $P$. vespertilionis dont les fréquences sont de $59 \%$ et de $9 \%$, et les densités de 4,0 et 2,0 , respectivement chez $R$. ferrum-equinum et $R$. euryale. Quant à $M$. peregrinus présent avec une fréquence de $15 \%$ chez $R$. ferrum-equinum, il n'a pas été rencontré chez $R$. euryale.

\section{b) Nématodes :}

Nous avons observé deux espèces de Nématodes chez $R$. ferrum-equinum : S. glycirrhiza et $L$. beaucournui; cette dernière seule a été récoltée chez $R$. euryale. La fréquence de $S$. glycirrhiza chez $R$. ferrum-equinum est très élevée (72\%), de sorte que son absence chez $R$. euryale, prend une signification particulière. La fréquence de $L$. beaucournui n'est pas identique chez les deux hôtes; $14 \%$ chez $R$. ferrumequinum et $24 \%$ chez $R$. euryale; c'est le seul de tous les parasites pour lequel la fréquence est plus élevée chez $R$. euryale.

\section{c) Cestodes :}

Chez $R$. ferrum-equinum, la fréquence de $H$. grisea atteint $19 \%$ et sa densité moyenne 13,0. Nous n'avons jamais rencontré de Cestode chez $R$. euryale. 


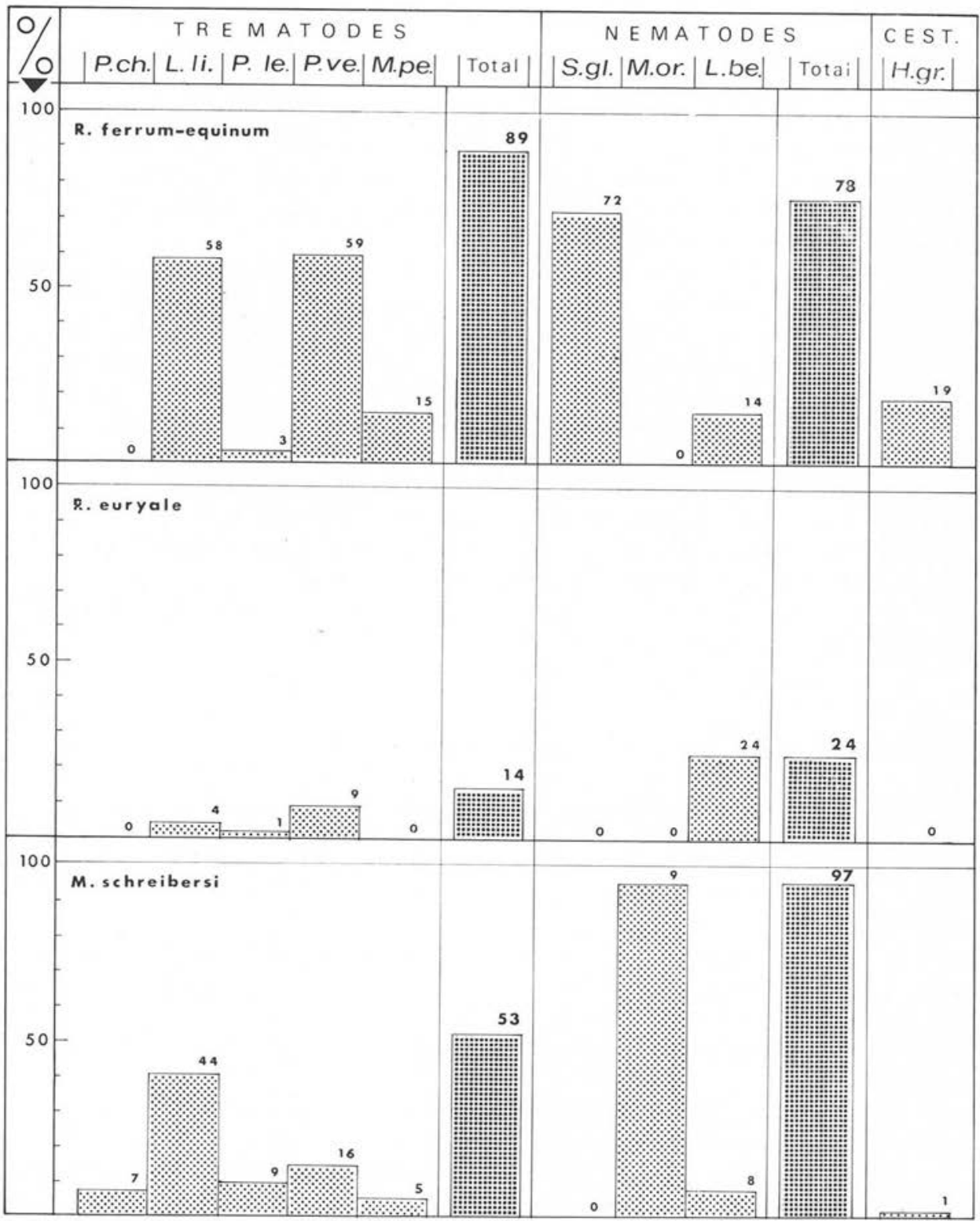

Fig. 9. - Comparaison de l'infestation chez Rhinolophus ferrum-equinum, Rhinolophus euryale et Miniopterus schreibersi 
Nous sommes en présence d'un problème d'écologie parasitaire tout à fait caractéristique ; deux espèces de Chauves-Souris très voisines par leur morphologie comme, semble-t-il, par leur biologie et leur écologie, présentent un parasitisme très différent : $R$. ferrum-equinum est une espèce toujours fortement parasitée, à l'inverse de ce qui s'observe chez $R$. euryale. On peut dire que dans notre région il est possible, sur un lot suffisant, de les distinguer par des statistiques parasitologiques. Nos résultats rappellent beaucoup ceux de Mituch (1964), qui note une helminthofaune très appauvrie chez $R$. euryale en Tchécoslovaquie (14\% de $R$. euryale parasités par des Helminthes contre $43 \%$ de $R$. ferrum-equinum).

On peut se demander si $R$. euryale ne présenterait pas une immunité totale (cas du Nématode $S$. glycirrhiza) ou partielle (cas des Trématodes en général), vis-à-vis des parasites. Toutefois, dans le cas des Trématodes, il faut envisager qu'un facteur de comportement trophique puisse intervenir: les connaissances sur l'éthologie de ces Rhinolophidés ne sont pas suffisamment détaillées pour que l'on puisse établir des corrélations entre le parasitisme et tel ou tel facteur de comportement, mais on peut supposer que les différentes espèces n'ont pas des mœurs (heures de sorties, milieux terrestres survolés, sélection des proies) identiques. Une étude des contenus stomacaux pourrait révéler des différences dans le pourcentage d'Insectes à larves aquatiques, vecteurs essentiels des Trématodes; on sait en effet que certaines espèces de Chiroptères choisissent un type de proies déterminé (Holmes, 1966).

La recherche précise de ces causes présenterait un grand intérêt pour l'écologie parasitaire de ces animaux.

\section{Caractéristiques de l'infestation chez $M$. schreibersi.}

Nos observations montrent que les $M$. schreibersi sont des Chauves-Souris fortement parasitées. Les espèces d'Helminthes $\mathrm{y}$ sont nombreuses et abondamment représentées.

\section{a) Trématodes :}

En plus des espèces rencontrées chez les Rhinolophidés, L. linstowi, P. lepidotus, $P$. vespertilionis, $M$. peregrinus, nous avons rencontré deux représentants du genre Prosthodendrium, $P$. chilostomum et $P$. parvouterus.

Pour l'ensemble des Trématodes, la fréquence est de $52 \%$, la densité moyenne de 13,4 .

Les $L$. linstowi sont rencontrés avec une fréquence et une densité élevées: $40 \%$ et 14,9 ; ces résultats sont comparables à ceux que nous avons notés chez $R$. ferrum-equinum $(58 \%$ et 20,6). P. vespertilionis est moins abondant: $14 \%$ et 2,1 . Les autres Trématodes sont plus rares.

b) NÉmATODEs :

Les Nématodes parasitent $95 \%$ des $M$. schreibersi; il s'agit de $M$. ornatus et de L. beaucournui. La fréquence de $M$. ornatus $(95 \%)$ est la plus élevée que nous ayons 
rencontrée au cours de nos recherches; cependant cette fréquence est approchée par celle de $S$. glycirrhiza chez $R$. ferrum-equinum. Il faut encore noter que $M$. ornatus a été observé chez Myotis myotis et $M$. capaccinii, mais jamais chez les Rhinolophidés. Enfin, L. beaucournui est présent mais avec une fréquence plus faible que chez les Rhinolophidés.

c) Cestodes :

Nous ne signalons qu'un cas d'infestation par les Cestodes $(H$. grisea) sur les 74 M. schreibersi disséqués, mais l'infestation était massive (plus de 100 individus).

Nos observations peuvent être comparées avec celles de Théodoridès (1953) qui a examiné la parasitofaune de $36 \mathrm{M}$. schreibersi récoltés dans la grotte de Pouade près de Banyuls-sur-Mer, au mois de mars. Cette station est située à quelques dizaines de kilomètres des localités que nous avons nous-mêmes prospectées. Théodoridès cite un Trématode ( $P$. vespertilionis) et deux Nématodes $(M$. ornatus et $L$. filaria). Si l'on tient compte qu'à l'époque du travail de Théodoridès, l'espèce beaucournui n'avait pas été séparée de l'espèce filaria, on constate que les trois parasites recueillis par Théodoridès ont été trouvés au cours de nos recherches; il est par contre remarquable qu'une espèce aussi commune que $L$. linstowi soit absente de la statistique de Théodoridès; la saison de la récolte n'est pas en cause car nos prélèvements de $M$. schreibersi réalisés au mois de mars nous ont livré des effectifs importants de L. linstowi.

Nous pensons qu'il serait particulièrement intéressant que des recherches parasitologiques soient poursuivies sur le Minioptère de Schreiber, car cette espèce possède une aire de distribution extrêmement vaste à l'intérieur de laquelle il «ne se localise que là où il trouve son biotope d'élection, si bien que les colonies peuvent être isolées les unes des autres par des vides de centaines de kilomètres » (Brosset, 1966). Lorsque serait connue la variation de sa parasitofaune non seulement dans l'espace mais aussi dans le temps des différences comme celles dont fait état la comparaison précédente pourraient vraisemblablement s'expliquer. On peut noter dès maintenant que diverses espèces, Prosthodendrium ascidia (Van Beneden, 1873), Parabascus duboisi (Hurkova, 1961), Moedlingeria amphoraeformis (Mödlinger, 1930), Ophiosacculus mehelyi (Mödlinger, 1930), Pycnoporus megacotyle (Ogata, 1938) signalées en Europe centrale par Hurkova (1963) et Matskasi $(1967,1968)$ ne semblent pas parasiter le Minioptère de Schreiber en Europe Occidentale.

\section{Observations sur l'infestation des autres espèces de Chiroptères.}

Les autres espèces de Chauves-Souris ont été trouvées en trop faible quantité pour espérer faire de nombreuses comparaisons sur leur parasitisme.

Cependant, nous pouvons noter certaines observations:

Toutes les espèces de Chauves-Souris abritaient Lecithodendrium linstowi sauf Rhinolophus hipposideros (6 individus examinés). L'infestation la plus massive a été observée chez Eptesicus serotinus (215 et 521 spécimens de L. linstowi respective- 
ment rencontrés chez les deux individus disséqués) et chez un Myotis capaccinii (205 L. linstowi).

Chez les Myotis myotis disséqués, nous n'avons rencontré qu'une seule espèce de Trématode: L. linstowi. Il est d'ailleurs présent avec une densité faible: les quatre Myotis myotis parasités renfermaient respectivement 2, 1, 1, 1 L. linstowi. Myotis myotis ncts a également livré ces Cestodes (1 individu sur 10 était parasité par Hymenolepis grisea) et des Nématodes (deux individus étaient parasités par Molinostrongylus ornatus).

Les Myotis emarginatus nous ont livré $P$. vespertilionis et $L$. linstowì.

L'un des Myotis capaccinii présentait 25 Lecithodendrium granulosum, espèce que nous n'avons rencontrée chez aucune autre Chauve-Souris.

Les Pipistrellus pipistrelius sont des Chauves-Souris fortement parasitées dans notre région. Les six individus étudiés étaient tous parasités par $L$. linstowi, avec une densité moyenne de 15,6. Il faut noter que Pipistrellus pipistrellus nous a livré le seul exemplaire de Fycnoporus heteroporus rencontré au cours de cette étude. Nous n'avons pas observé la présence de Cestodes ou de Nématodes.

Enfin, l'un des Plecotus austriacus disséqués abritait $L$. linstowi et $P$. lepidotus.

Le tableau $\mathrm{V}$ résume l'ensemble des données qualitatives sur le parasite des Chiroptères dans la région envisagée.

\section{Tableau V}

\begin{tabular}{|c|c|c|c|c|c|c|c|c|c|c|c|c|c|c|}
\hline & 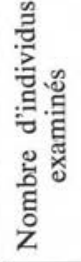 & 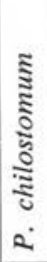 & 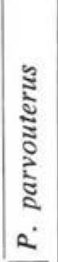 & 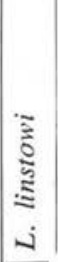 & 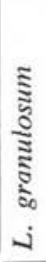 & $\begin{array}{l}3 \\
\vdots \\
\vdots \\
\vdots \\
\vdots \\
\vdots \\
\vdots \\
2 \\
2\end{array}$ & 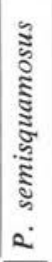 & 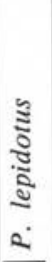 & 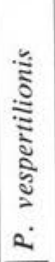 & 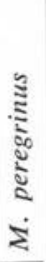 & 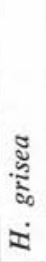 & 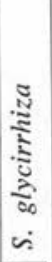 & 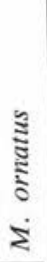 & 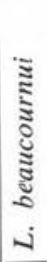 \\
\hline $\begin{array}{l}\text { Rhinolophus ferrum-equi- } \\
\text { num } \quad . . \ldots \ldots \ldots \ldots \ldots\end{array}$ & 96 & & & $*$ & & & & $*$ & * & ${ }^{*}$ & * & $*$ & & $*$ \\
\hline Rhinolophus euryale ..... & 80 & & & *1 & & & & * & $*$ & & & & & $*$ \\
\hline Rhinolophus hipposideros & 6 & & & & & & & & * & $*$ & & & & \\
\hline Miniopterus schreibersi .. & 74 & $*$ & $*$ & $*$ & & & & * & * & $*$ & $*$ & & $*$ & * \\
\hline Myotis myotis .......... & 18 & & & $*$ & & & & & & & $*$ & & * & \\
\hline Myotis capaccinii ....... & 7 & & & $*$ & $*$ & & & & $*$ & & & & & \\
\hline Myotis emarginatus ..... & 2 & & & $*$ & & & & & $*$ & & $*$ & & & * \\
\hline Eptesicus serotinus ...... & 2 & & & $*$ & & & & & * & & & & & \\
\hline 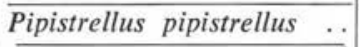 & 6 & & & $*$ & & $*$ & $*$ & & $*$ & & & & & \\
\hline Plerotus austriacus ...... & 2 & & & $*$ & & & & $*$ & & & & & & \\
\hline
\end{tabular}




\section{Caractéristiques de l'infestation en fonction du milieu}

Les Chiroptères que nous avons examinés ont été capturés soit dans des biotopes naturels (grottes), soit dans des biotopes artificiels (souterrains, constructions diverses) ; il est probable qu'une étude approfondie de ces deux types de milieux révélerait de notables différences dans les facteurs biotiques (faune associée notamment) et abiotiques (humidité, température) qui y règnent. Il est donc intéressant de rechercher si le parasitisme des Chauves-Souris ne pourrait représenter un indicateur valable de l'habitat habituel des hôtes.

Pour deux espèces, $R$. ferrum-equinum et $M$. schreibersi nous avons disposé d'effectifs, sinon équivalents, du moins non négligeables provenant de grottes et provenant de souterrains.

Les tableaux VI et VII indiquent les caractéristiques du parasitisme de ces deux Chiroptères d'après l'habitat où ils ont été capturés.

\section{Tableau VI}

Rhinolophus ferrum-equinum

\begin{tabular}{|c|c|c|}
\hline & $\begin{array}{c}\text { Grottes } \\
(23 \text { individus })\end{array}$ & $\begin{array}{l}\text { Souterrains } \\
\text { (73 individus) }\end{array}$ \\
\hline$P$. vespertilionis $\ldots \ldots \ldots \ldots \ldots$ & $57 \%$ & $51 \%$ \\
\hline M. peregrinus $\ldots \ldots \ldots \ldots \ldots \ldots$ & 0 & $24 \%$ \\
\hline L. linstowi $\ldots \ldots \ldots \ldots \ldots \ldots$ & $\Delta \cap \%$ & $67 \%$ \\
\hline P. lepidotus $\ldots \ldots \ldots \ldots \ldots \ldots$ & 0 & $4 \%$ \\
\hline Total Trématodes...$\ldots \ldots \ldots$ & $81 \%$ & $92 \%$ \\
\hline H. grisea (Cestodes) ............. & $19 \%$ & $16 \%$ \\
\hline S. glycirrhiza $\ldots \ldots \ldots \ldots \ldots$ & $14 \%$ & $89 \%$ \\
\hline L. beaucournui .............. & 0 & $17 \%$ \\
\hline Total Nématodes...$\ldots \ldots \ldots$ & $14 \%$ & $91 \%$ \\
\hline
\end{tabular}

Ces tableaux montrent, surtout pour les Trématodes, qu'aucune différence significative ne semble se révéler. Si les fréquences de chacune des espèces de parasites ne sont évidemment pas identiques, il apparaît clairement que le taux moyen d'infestation par les Trématodes atteint un niveau très semblable. La seule différence notable (le calcul du $\%^{2}$ indique qu'elle est significative à $1 \%$ ) concerne le nématode S. glycirrhiza; si cette différence était confirmée, nous pensons qu'elle permettrait, dans une population de Rhinolophes donnée, de distinguer les fractions de cette popu- 
Tableau VII

Miniopterus schreibersi

\begin{tabular}{|c|c|c|}
\hline & $\begin{array}{c}\text { Grottes } \\
\text { (37 individus) }\end{array}$ & $\begin{array}{l}\text { Souterrains } \\
\text { ( } 37 \text { individus) }\end{array}$ \\
\hline P. vespertilionis $\ldots \ldots \ldots \ldots \ldots$ & $16 \%$ & $16 \%$ \\
\hline M. peregrinus $\ldots \ldots \ldots \ldots \ldots$ & $8 \%$ & $3 \%$ \\
\hline L. linstowi $\ldots \ldots \ldots \ldots \ldots \ldots$ & $35 \%$ & $54 \%$ \\
\hline P. lepidotus ............... & $3 \%$ & $16 \%$ \\
\hline P. chilostomum $\ldots \ldots \ldots \ldots \ldots$ & $13 \%$ & 0 \\
\hline P. parvouterus $\ldots \ldots \ldots \ldots \ldots$ & $5 \%$ & 0 \\
\hline Total Trématodes ........... & $57 \%$ & $54 \%$ \\
\hline H. grisea (Cestodes) .......... & 0 & $3 \%$ \\
\hline M. ornatus $\ldots \ldots \ldots \ldots \ldots$ & $97 \%$ & $97 \%$ \\
\hline L. beaucournui $\ldots \ldots \ldots \ldots \ldots$ & $8 \%$ & $11 \%$ \\
\hline Total Nématodes $\ldots \ldots \ldots \ldots$ & $97 \%$ & $97 \%$ \\
\hline
\end{tabular}

lation qui seraient plus ou moins fidèles à un biotope diurne d'un type déterminé ; cette hypothèse n'est pas en contradiction avec ce que l'on connaît de la biologie de $R$. ferrum-equinum.

En comparant de façon détaillée les prélèvements effectués dans chaque localité, nous avons noté de plus que ce sont les Chiroptères du Château de Salses qui paraissent les plus riches en Trématodes. L'environnement de cette localité comprend des canaux d'irrigation, ruisseaux et marécages riches en Mollusques et très favorables au déroulement des cycles.

Si l'on met à part le cas de $S$. glycirrhiza, la ressemblance du parasitisme des animaux capturés dans les grottes et les souterrains est cependant très grande; nous ne pensons pas que cette observation, à propos de Chiroptères, constitue un fait étonnant, et cela pour deux raisons :

a) d'une part, les Chauves-Souris étudiées ne sont nullement sédentaires à l'échelle de l'habitat: tel individu capturé dans une grotte, pouvait se trouver récemment dans un souterrain et vice versa.

b) d'autre part, les cycles d'Helminthes de Chiroptères se déroulent en majorité «à l'extérieur» et non dans l'environnement très particulier des grottes ou des souterrains. La faune associée des milieux hypogés n'est donc pas un facteur primordial. Les facteurs biotiques expliquant la présence des parasites, sont à rechercher dans le 


\section{Tableau VIII}

\section{Rhinolophus ferrum-equinum}

Salses, 17-11-67. Statistique portant sur 15 individus.

\begin{tabular}{|c|c|c|c|c|c|c|c|c|c|c|}
\hline & P.ve. & M. pe. & L.li. & P. ch. & P.le. & $\begin{array}{l}\text { Total } \\
\text { Trém. }\end{array}$ & $\begin{array}{l}H . g r . \\
\text { (Cest.) }\end{array}$ & S. gl. & L. be. & $\begin{array}{l}\text { Total } \\
\text { Ném. }\end{array}$ \\
\hline d. & 4 & 0 & 9 & 0 & 4 & - & 12 & 5 & 0 & - \\
\hline F. & $60 \%$ & $0 \%$ & $53 \%$ & $0 \%$ & $6 \%$ & $86 \%$ & $13 \%$ & $86 \%$ & $0 \%$ & $86 \%$ \\
\hline
\end{tabular}

Salses, 15-3-68. Statistique portant sur 19 individus.

\begin{tabular}{|c|c|c|c|c|c|c|c|c|c|c|}
\hline & P. ve. & M. pe. & L.li. & P. ch. & P.le. & $\begin{array}{l}\text { Total } \\
\text { Trém. }\end{array}$ & $\begin{array}{l}H . g r . \\
\text { (Cest.) }\end{array}$ & S. gl. & L. be. & $\begin{array}{l}\text { Total } \\
\text { Ném. }\end{array}$ \\
\hline d. & 4 & 3 & 29 & 0 & 0 & - & 4 & 9 & 3 & - \\
\hline F. & $47 \%$ & $36 \%$ & $34 \%$ & $0 \%$ & $0 \%$ & $94 \%$ & $21 \%$ & $94 \%$ & $26 \%$ & $94 \%$ \\
\hline
\end{tabular}

Salses, 13-4-68. Statistique portant sur 20 individus.

\begin{tabular}{|c|c|c|c|c|c|c|c|c|c|c|}
\hline & P. ve. & M. pe. & L. li. & P. ch. & P.le. & $\begin{array}{l}\text { Total } \\
\text { Trém. }\end{array}$ & $\begin{array}{l}\text { H. gr. } \\
\text { (Cest.) }\end{array}$ & S. $g l$. & L. be. & $\begin{array}{l}\text { Total } \\
\text { Ném. }\end{array}$ \\
\hline d. & 3 & 2 & 15 & 0 & 0 & - & 3 & 6 & 1 & - \\
\hline F. & $70 \%$ & $15 \%$ & $5 \%$ & $0 \%$ & $0 \%$ & $90 \%$ & $15 \%$ & $90 \%$ & $30 \%$ & $95 \%$ \\
\hline
\end{tabular}

Salses, 11-5-68. Statistique portant sur 11 individus.

\begin{tabular}{|c|c|c|c|c|c|c|c|c|c|c|}
\hline & P. ve. & M. pe. & L. li. & P. ch. & P.le. & $\begin{array}{l}\text { Total } \\
\text { Trém. }\end{array}$ & $\begin{array}{l}H . g r . \\
\text { (Cest.) }\end{array}$ & S. gl. & L. be. & $\begin{array}{l}\text { Total } \\
\text { Ném. }\end{array}$ \\
\hline d & 5 & 1 & 22 & 0 & 1 & - & 30 & 3 & 1 & - \\
\hline F. & $72 \%$ & $18 \%$ & $63 \%$ & $0 \%$ & $18 \%$ & $100 \%$ & $27 \%$ & $72 \%$ & $9 \%$ & $72 \%$ \\
\hline
\end{tabular}


milieu extérieur que fréquentent habituellement les Chiroptères. Il est bon de rappeler que la quasi-totalité de la nourriture de ces animaux est prise à l'extérieur.

\section{Caractéristiques de l'infestation en fonction de la saison}

Les observations dans ce domaine sont délicates, car à certains moments de lannée, nous avons éprouvé des difficultés pour localiser les colonies des espèces étudiées. Pour $R$. ferrum-equinum, nous pouvons donner une statistique répartie au cours de l'année. Cette statistique (tableau VIII) montre qu'il n'existe pas de différence très nette dans le parasitisme suivant la date des prélèvements.

En particulier, au moment de l'hibernation, bien que la faune des Trématodes paraisse diminuer légèrement, on ne peut pas parler d'une défaunation hivernale caractéristique. Les prélèvements de janvier à mars ayant été faits au moment même de l'hibernation de l'hôte, alors que la température de celui-ci est fortement diminuée, il est certain que la majorité des Helminthes de ce Rhinolophe s'accommodent parfaitement d'une importante hypothermie.

$$
\text { ** }
$$

\section{Bibliographie}

ANdReiko (O. F.) et Skvortsov (V. G.), 1967. - La faune helminthologique des Chiroptères de Moldavie et son écologie. Problemy Parazit: 130-132 (en russe).

Bhalerao (G. D.), 1926. - The intestinal parasites of the bat (Nyctinomus plicatus) with a list of the Trematodes hitherto recorded from Burma. J. Burma Res. Soc. Rangoon, 15 (3) : 181-195.

Caballero (E.), 1960. - Trematodes de los Murcielagos de Mexico. Catalogo Taxonomico de los Trematodos que parasitan a los Murcielagos (Mammalia, Chiroptera Blumenbach, 1774). An. Instit. Biol., 31 (1, 2) : 215-287.

DAwEs (B.), 1956. - The Trematoda, with special reference to British and other European forms. Cambridge Univers. Press. Edit. : 644 p.

Dollfus (R.-Ph.), 1954. - Miscellanea Helminthologica Maroccana XVI. Sur un Distome de Microchiroptère. Arch. Inst. Pasteur Maroc, 4 (9) : 625-635.

-, et coll., 1961. - Station expérimentale de Parasitologie de Richelieu (Indre-et-Loire). Contribution à la faune parasitaire régonale. Ann. Parasitol., 36 (3) : 169-451.

—, 1968. - Les Trématodes de l'Histoire Naturelle des Helminthes de Félix Dujardin (1845). Mém. Mus. Nat. Hist. nat., Sér. A, Zool., 54 (3) : 119-196.

Dubcis (G.), 1955. - Les Trématodes de Chiroptères de la collection Villy Aellen., Rev. Suisse zool., 62 (3) : 469-506.

—, 1956. - Contribution à l'étude des Trématodes de Chiroptères. Rev. Suisse Zool., 63 (4) : 683-695. 
—, 1960. - Contribution à l'étude des Trématodes de Chiroptères. Rev. Suisse Zool., 67 (1) : $1-80$.

Dujardin (F.), 1845. - Histoire naturelle des Helminthes ou Vers intestinaux. Paris: 654 p. Holmes (J.-C.), 1966. - Factors influencing the Trematode fauna of bats. Proc. first. intern. Congr. Parasitol., Vol. I : 490-492.

Hurkova (J.), 1963. - Bat Trematodes in Czechoslovakia. I. A systematical review of occuring species. Vest. Cs. spol. zool. (Acta soc. zool. Bohemoslov.), 27 (4) : 250-276.

Liang Sheng (Y.), 1957. - Studies on a Trematode and a New Nematode from a Bat from Northern Rhodesia. J. Helminthol., 31: 121-125.

LuHe (M.), 1961. - Trematodes. In: Die Susswasserfauna Deutschlands, 17: 217 p.

Matsaberidze (G. V.) et Khotenovski (I. A.), 1967. - Trématodes de Chiroptères de Géorgie (in: Helminthes d'animaux et plantes de Géorgie). Tibilisi : Izdatelokvo Metsniereba : 83-94 (en russe).

Matskasi (I.), 1967. - The Systematico-Faunistical Survey of the Trematode Fauna of Hungarian Bats. I. Ann. Histor. Nat. Mus. nat. Hungarici (Pars Zool.), 59: 217-238.

Mituch (J.), 1964. - Contribution to the Knowledge of the Helminih Fauna in Bats of the Family Rhinolophidae in Slovakia (C.S.S.R.). Helminthologia., $5: 33-48$.

-, 1965. - Beitrag zur Erkenntnis der Helminthenfauna von Miniopterus schreibersii (Kuhl, 1819) in der Slowakei (C.S.S.R.). Helminthologia., 6 (1/4) : 109-119.

OdEning (K.), 1964. - Erkretionssystem und systematische Stellung einiger Fledermaustrematoden aus Berlin und Umgebung nebst Bemerkungen zum Lecithodendrioiden Komplex. Z. Parasitenk., 24 : 453-483.

Salvayre (H.), 1962. - Essai sur l'étude des groupements de Chauves-Souris dans le Conflent (I). Conflent, 2 (8) : 59-62.

—, 1962. - Essai sur l'étude des groupements de Chauves-Souris dans le Conflent (II). Conflent, 2 (9): 104-113.

—, 1965. - Inventaire spéléologique du Synclinal de Merens à Villefranche (Pyrénées-Orientales). Bull. Soc. Sci. Agr. Litt. Pyr.-Or.: 73-91.

Soltys (A.), 1959. - The Helminth Fauna of Bats (Chiroptera) of Lublin Palatinate. Acta parasitol. polon., $7: 599-613$.

Stiles (C. W.) et Mabelle (O. N.), 1930. - Key Catalogue Reported for Chiroptera (Bats) with their Possible Public Health Importance. Nat. Inst. Health. Bull., 153 : 603-742.

THÉODORIDÈs (J.), 1953. - Statistique du parasitisme par Helminthes chez Miniopterus schreibersi Natt. (Cheiroptera, Vespertilionidae). Vie et Milieu., 4 (1) : 128-129.

Timon-David (J.), 1964. - Contribution à la connaissance des Helminthes du Rhinolophe fer à cheval en Provence. Vie et Milieu, 15 (1) : 139-151.

Vaucher (C.) et Hunkeler (P.), 1967. - Contribution à l'étude des Cestodes et des Trématodes parasites des Micromammifères de Suisse. I. Bull. Soc. neuchâtel. Sci. nat., $90: 161-184$.

YAmaguti (S.) et Asada (Z.), 1942. - Trematodes of birds and mammals from Manchoukuo, I. Bull. Inst. of Scientific Research, Manchoukuo, 6 (4) : 498-512. 